\title{
CHEGOU A ERA DA INTERNET DAS COISAS? UM ESTUDO SOBRE ADOÇÃO DE OBJETOS INTELIGENTES NO CONTEXTO BRASILEIRO
}

Objetivo: Este estudo objetivou identificar as variáveis determinantes para adoção de "Objetos Inteligentes" com atributos da "Internet das Coisas", por parte de consumidores no contexto brasileiro.

Método: Para tanto, foram apresentados os conceitos e definições de "Internet das Coisas" e "Objetos Inteligentes" e identificadas as variáveis que podem influenciar a adoção deste tipo de tecnologia de acordo com referencial teórico, a saber: utilidade percebida, facilidade de uso, prazer percebido, influência social, confiança, controle comportamental percebido e custo, valendo-se de pesquisa quantitativa, com uma amostra de 208 pessoas e análise dos dados realizada através de Modelagem de Equações Estruturais.

Originalidade / relevância: É possível verificar que existem poucos estudos sobre a "Internet das Coisas" e sua influência sobre os consumidores brasileiros, embora o tema venha sendo discutido em diversos fóruns de tecnologia no país o que denota a importância da presente pesquisa para consolidação do conhecimento sobre o tema.

Resultados: Através desta pesquisa, verificou-se que as variáveis determinantes para adoção de "Objetos Inteligentes" na amostra estudada são: custo e controle comportamental.

Contribuições teóriocas/metodológicas: Tal resultado colabora para o enrequecimento do campo de conhecimento do comportamento do consumidor em um novo contexto tecnológico, e permite a replicação do modelo teórico utilizado em outros contextos.

Contribuições sociais/para a gestão: Contribui também para criação de estratégias mercadológicas mais assertivas considerando as preferencias do consumidor brasileiro no que diz respeito ao custo e ao senso de controle sobre os "Objetos Inteligentes".

Palavras-chave: Internet das Coisas. Comportamento do Consumidor. Adoção de Tecnologia. Objetos Inteligentes. Modelagem de Equações Estruturais.

\section{THE INTERNET OF THINGS AGE HAS ARRIVED? A STUDY ABOUT THE ADOPTION OF SMART OBJECTS IN THE BRAZILIAN CONTEXT}

Objective: This study aimed to identify the determinant variables to the adoption of "Smart Objects" with "Internet of Things" attributes, by consumers in the Brazilian context.

Method: For this, it were presented the concepts and definitions of the terms "Internet of Things (IoT)" and "Smart Objects" and identified the variables that can influence the adoption of this kind of technology according to theoretical research, namely: perceived usefulness, perceived ease of use, perceived enjoyment, social influence, trust, perceived behavioral control and cost, considering a quantitative research, with a sample of 208 people, and data analysis using the Structural Equations Modeling.

Originality / relevance: It is possible to verify that there are just few studies about the "Internet of Things" and its influence on the Brazilian consumers, although this theme has been discussed in several technology forums around the country, what highlights the importance of the present research to consolidate this theme's knowledge.

Results: Through this research, it was verified that, to the analyzed sample, the determinant factors to the adoption of "Smart Objects" are: cost and perceived behavioral control.

Theoretical / Methodological contributions: Such result collaborates to the enrichment of the consumer's behavior knowledge in a new technological context, and enables the replication of the theoretical model used, in other contexts.

Social / Managerial contributions: It also contributes to the creation of more assertive market strategies considering the Brazilian consumers preferences regarding the aspects: consumer's sense of control and the cost of "Smart Objects".

Keywords: Internet of Things. Consumer Behavior. Technology Adoption. Smart Objects. Structural Equations Modeling.

Gabriela da Silva Gomes ${ }^{1}$ Fabio Vinicius de Macedo Bergamo ${ }^{2}$

\footnotetext{
${ }^{1}$ Graduanda em Administração pelo Centro Universitário Adventista de São Paulo - UNASP. São Paulo, Brasil. E-mail: gabrielasilva_gomes@yahoo.com.br

${ }^{2}$ Doutor em Administração pela Universidade Federal da Bahia - UFBA. Professor no Centro Universitário Adventista de São Paulo - UNASP. São Paulo, Brasil. E-mail: fabio.bergamo@ unasp.edu.br
} 


\section{INTRODUÇÃO}

Os objetos não são mais os mesmos. Por meio dos avanços tecnológicos, o relógio que antes apenas mostrava as horas, agora pode receber ligações, notificações de redes sociais e e-mails além de acompanhar dados sobre a saúde de seu usuário, e a geladeira que unicamente conservava alimentos, agora pode também controlar prazos de validade e fazer compras. Graças a uma nova convergência da internet, denominada "Internet das Coisas", utensílios, acessórios e uma série de outras "coisas" podem se conectar a rede, interagir entre si e executar suas funções sem a necessária intervenção e controle daquele faz uso delas (Lemos, 2013).

Com novos atributos e funções, os objetos estão cada vez mais inteligentes e capazes de executar desde as atividades mais básicas do dia-adia, como limpar e cozinhar, até as mais complexas, como monitorar o tráfego de veículos de uma cidade inteira. Por esse motivo a adoção de produtos com essas características tem se tornado notável considerando a estimativa de que, até 2026, o número de objetos conectados à internet no mundo seja superior à população mundial atual em aproximadamente 14 vezes, o que representa mais de 100 bilhões de relógios, televisões, geladeiras, casas, carros e afins, comunicando-se uns com os outros e com o ambiente que os cerca através da internet (Serviço Brasileiro de Apoio às Micro e Pequenas Empresas [SEBRAE], 2016, 05 de Janeiro).

As funções dos objetos no contexto da "Internet das Coisas", e as suas diversas aplicações e implicações no dia-a-dia das pessoas individualmente e da sociedade como um todo, têm sido alvos de discussões principalmente nos últimos anos, isso porque, embora haja uma série de benefícios práticos na adoção desses "Objetos Inteligentes", existem sérias preocupações quanto à proteção de dados pessoais e o constante controle e monitoramento da vida de seus usuários (Isaia, 2016). Entretanto, embora o tema tenha ganhado destaque no Brasil, existem poucos estudos científicos no país que versem sobre os reais impactos que esse novo momento tecnológico pode trazer a sociedade e sobre como esta responderá a tais mudanças. Dentro da área de Marketing, há uma necessidade de melhor entendimento de como é a aceitação e o comportamento de consumo de “objetos inteligentes", haja vista que os mesmos são identificados como tecnologias que podem alterar a forma como o consumidor se relaciona com marcas e empresas, principalmente no varejo e nas relações mediadas por plataformas sociais (Atzori, Iera \& Morabito, 2014; Lee \& Lee, 2015; Bergamo, 2017).

Levando em conta tais considerações, e a crescente oferta e demanda de novas tecnologias no mercado, faz-se necessário entender quais são as principais variáveis consideradas pelo consumidor de tecnologia no contexto da "Internet das Coisas", para adoção de produtos tecnológicos. Em outras palavras, as organizações que produzem ou pretendem produzir "Objetos Inteligentes", precisam compreender as principais variáveis que seriam consideradas pelos consumidores ao adotar tais produtos.

Nesse sentido, o presente estudo objetivou responder ao seguinte questionamento: quais as variáveis determinantes no processo de adoção de "Objetos Inteligentes" por parte dos consumidores no contexto brasileiro, no que diz respeito à "Internet das Coisas"? Para tanto, este estudo buscou conceituar "Internet das Coisas" e "Objetos Inteligentes"; identificar quais são as principais variáveis influenciadoras da aceitação de tecnologia baseada na "Internet das Coisas" por meio de revisão teórica; e verificar quais dessas variáveis são determinantes para a adoção dos "Objetos Inteligentes" por parte dos consumidores no contexto brasileiro.

\section{REVISÃO TEÓRICA}

\subsection{A "Internet das Coisas": Conceito e Definições}

O termo "Internet das Coisas" foi utilizado pela primeira vez em 1999 por Kevin Ashton em uma palestra a respeito do uso de etiquetas de radiofrequência (comunicação entre um objeto e outro, via ondas de rádio) ou RFID (RadioFrequency IDentification) e sobre como essa tecnologia poderia revolucionar a relação entre os seres humanos e os objetos e sobre as facilidades que a mesma poderia trazer para as rotinas organizacionais (Lemos, 2013). Este termo representa a capacidade de comunicação entre objetos por meio da internet, o que possibilita a criação de diversas soluções no âmbito doméstico, nas áreas de lazer, construções, entre outras, para o dia-a-dia das pessoas (Kadow \& Camargo, 2016).

Entretanto, mais do que uma nova nomenclatura que define um momento singular da constante evolução tecnológica, a "Internet das Coisas" representa o futuro das empresas no que diz respeito às estratégias mercadológicas, das interações humanas no âmbito social, e até mesmo nas formas de consumo (Segura \& Hildebrand, 2014).

Considerando a proporção dos impactos e implicações trazidas à luz pelo advento de uma era hiperconectada tal qual a vivenciada hoje, é possível compreender porque este assunto vem sendo cada vez mais discutido e noticiado (Singer, 2013). Além 
disso, inúmeras pesquisas científicas têm sido realizadas na tentativa de compreender de que maneira as pessoas, as empresas e a sociedade em si serão afetadas pela "Internet das Coisas" e qual será a sua reação. Gao e Bai (2014), por exemplo, buscaram compreender aspectos comportamentais de consumidores chineses frente à utilização de um serviço baseado na "Internet das Coisas", enquanto Kadow e Camargo (2016) objetivaram averiguar questões referentes à segurança e privacidade de dados dos usuários dessa tecnologia. Al-momani, Mahmoud e Ahmad (2016), por sua vez, buscaram delinear um modelo conceitual levando em conta algumas das diversas variáveis que podem ser consideradas pelos consumidores de tecnologia no contexto da "Internet das Coisas".

Percebe-se, portanto, que essa nova configuração tecnológica é uma realidade, um fenômeno que precisa ser compreendido, bem como a sua influência em todos os aspectos da vida do ser humano e de sua relação com a tecnologia.

\subsection{Da internet a "Internet das Coisas"}

Embora sondar aspectos técnicos e estruturais da internet não seja o objetivo central desta pesquisa, faz-se necessário considerar, de maneira sucinta, a sua evolução ao longo do tempo até chegar ao modelo atual. Quando em seus primórdios, a internet representava uma conexão limitada entre um computador e o outro, ou seja, o fluxo de dados na rede entre o dispositivo emissor e o dispositivo receptor era proporcional e linear (Paes, 2014). Segundo Gubbi, Buyya, Marusic e Palaniswami (2013), com o passar do tempo, os fios outrora necessários desapareceram, dando lugar à mobilidade, e além disso, as barreiras de espaço e as limitações quanto à conexão deixaram de existir, e passou-se cada vez mais a utilizar chips, sensores, e tecnologias sem fio como o Wifi (Wireless Fidelity), Bluetooth e o RFID.

A possibilidade de atribuir uma identidade digital (IP - Internet Protocol) aos objetos permite que a internet esteja presente em todos os lugares e, por isso, conforme observado por Paes (2014), a "Internet das Coisas" representa um estado difuso da internet onde há interação irrestrita entre os objetos e as pessoas e, na medida em que a capacidade de conexão das "coisas" aumenta, o tráfego de dados na rede se torna proporcionalmente maior. Consequentemente, as informações e dados pessoais dos usuários podem sofrer o risco de ser constantemente monitoradas, trazendo à tona problemas de segurança e privacidade (Kadow \& Camargo, 2016), grande ponto de discussão do tema em questão.

\subsection{Os "Objetos Inteligentes"}

Conforme considerado anteriormente, a tecnologia evoluiu, a capacidade de conexão melhorou e a internet se transformou tornando-se menos limitada. Nesse sentido, por que esperar que os objetos continuassem os mesmos? Se por meio de um sensor ou acessório que permita sua conexão com a internet, um objeto pode fazer mais do que fazia, por que não agrega-lo à evolução tecnológica? Os objetos também evoluíram e, segundo Santaella, Gala, Policarpo e Gazoni (2013), passaram a ser mais ativos e presentes na vida das pessoas mudando o seu foco de atuação do auxílio ao homem para uma maior independência do mesmo.

Basicamente, o que diferencia um objeto comum de um objeto inteligente, é a capacidade deste de se conectar a internet. E quando um objeto pode se conectar a internet, consequentemente ele pode se comunicar com outros objetos também conectados, transmitir e enviar dados e interagir com o ambiente que o cerca respondendo aos seus incentivos (Paes, 2014). É nesse ponto que um relógio que apenas mostra as horas se diferencia de um relógio inteligente com as características já mencionadas neste artigo.

Nesse contexto, Lemos (2013) apresenta um cenário em que os objetos modificam a maneira das pessoas se relacionarem entre si e com as diversas variáveis que as cercam enquanto membros de uma sociedade. Isso porque, segundo Squirra (2016), se tais objetos se tornam mais funcionais, as pessoas tenderão a busca-los com maior intensidade com o objetivo de satisfazer às suas necessidades e, ao mesmo tempo em que os objetos adquirem maior autonomia de ação, os seres humanos tornam-se cada vez mais adeptos aos mesmos.

\section{VARIÁVEIS INFLUENCIADORAS DA ADOÇÃO DE PRODUTOS COM ATRIBUTOS DA "INTERNET DAS COISAS"}

A disposição dos consumidores em adotar novas tecnologias é influenciada por uma série de variáveis. Ao pesquisarem sobre a aceitação de serviços com atributos da "Internet das Coisas" por parte dos consumidores chineses, Gao e Bai (2014) consideraram as variáveis: utilidade percebida, facilidade de uso percebida, confiança, influência social, prazer percebido e controle comportamental percebido. Os autores combinaram dois modelos teóricos extensamente utilizados em pesquisas referentes à aceitação de tecnologia pelos consumidores, a saber, o TAM (Technology Acceptance Model) e o UTAUT (Unified Theory of Acceptance and Usage of Technology) e também incluíram outras variáveis que julgaram relevantes 
para sua pesquisa. Al-Momani, Mahmoud e Ahmad (2016), por sua vez, propuseram um modelo conceitual que também combina o TAM e o UTAUT, e levaram em conta outras considerações por meio de revisão teórica. Tal modelo conceitual considerava as seguintes variáveis: utilidade percebida, facilidade de uso percebida, influência social, custo, conhecimento em TI (tecnologia da informação), confiança, privacidade e segurança. Estes últimos, diferentemente de Gao e Bai (2014), não consideraram as variáveis prazer percebido e controle comportamental, mas sim custo, conhecimento em TI e privacidade e segurança.

Ao comparar ambos os modelos propostos pode-se verificar que existem variáveis equivalentes. A variável confiança é definida por Gao e Bai (2014) como sendo a diminuição da sensação de insegurança do indivíduo quanto ao uso de suas informações pessoais, além da credibilidade no que diz respeito ao bom funcionamento do produto. Enquanto isso, as variáveis privacidade e segurança são definidas por Al-Momani, Mahmoud e Ahmad (2016) como sendo os aspectos relativos à integridade pessoal de um indivíduo e do senso de domínio que este possui sobre suas informações particulares. É possível notar que a variável confiança proposta por Gao e Bai (2014) engloba indicadores relativos à privacidade e segurança, propostos no modelo de Al-Momani, Mahmoud e Ahmad (2016).

Situação semelhante ocorre com as variáveis controle comportamental percebido e conhecimento em TI. A primeira é definida por Gao e Bai (2014) como sendo sensação de aptidão que o indivíduo possui para utilizar um serviço ou produto com atributos tecnológicos, além do domínio das funções de tal produto ou serviço. A última é definida por Al-Momani, Mahmoud e Ahmad (2016) como a capacidade de um indivíduo de conhecer o funcionamento de um produto ou serviço com atributos tecnológicos, e saber como utilizá-lo. Percebe-se, então, que as definições são equivalentes.

Para fins deste estudo, serão consideradas as variáveis: confiança e controle comportamental percebido. Na Figura 1 é possível verificar o modelo proposto considerando a combinação de ambos os modelos acima citados, o qual proporciona uma análise mais abrangente das variáveis influenciadoras da adoção de inovações tecnológicas.

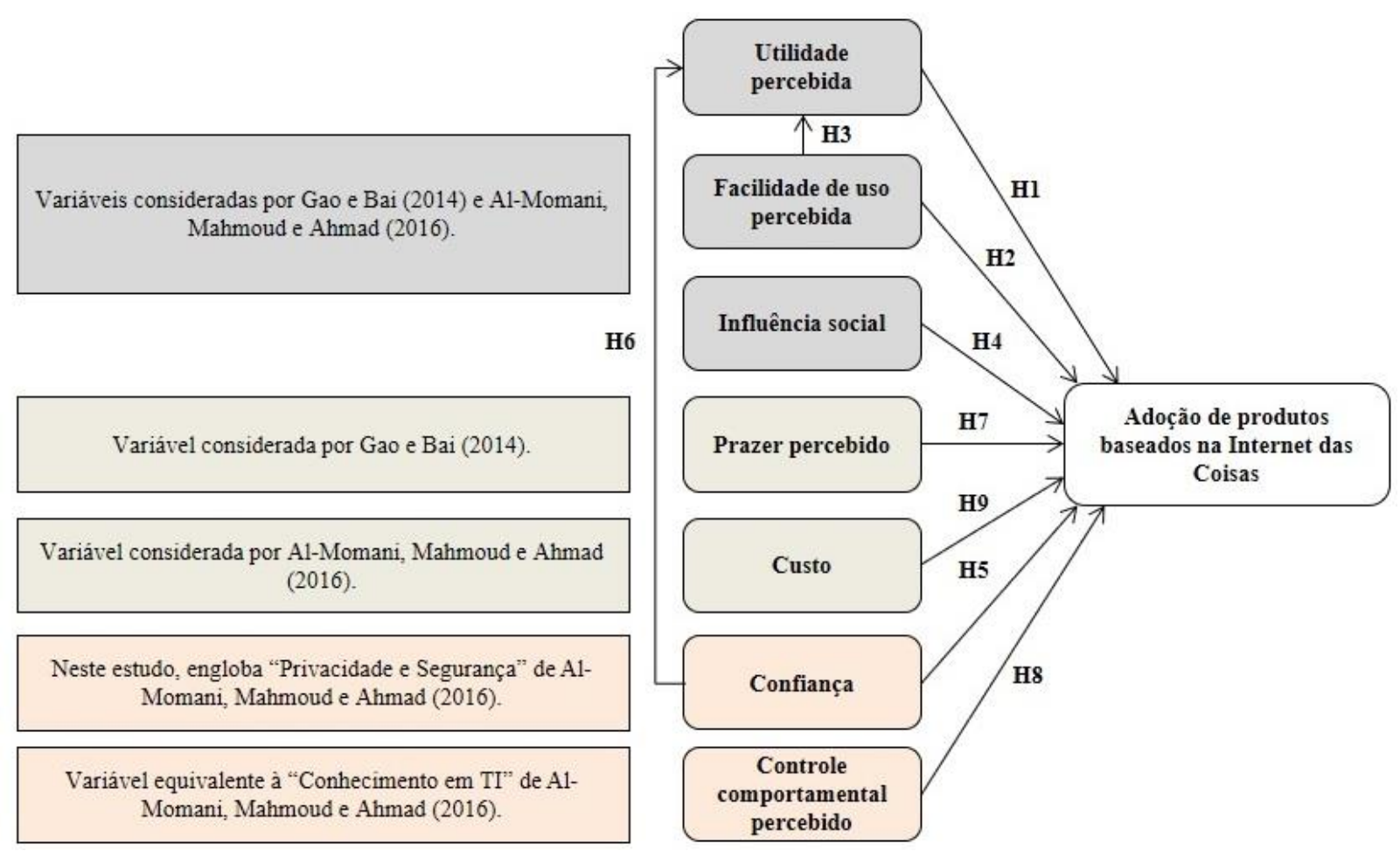

Figura 1 - Comparação dos modelos conceituais propostos por Al-Momani, Mahmoud e Ahmad (2016) e Gao e Bai (2014).

Fonte: Elaboração própria, em adaptação a Al-Momani, Mahmoud e Ahmad (2016) e Gao e Bai (2014). 
A definição de cada uma das variáveis consideradas de acordo com o modelo de análise proposto, bem como as hipóteses que nortearão a análise dos resultados, são expostas no quadro 1:

\begin{tabular}{|l|l|l|}
\hline Variável & Definição & $\begin{array}{l}\text { Referencial } \\
\text { Teórico }\end{array}$ \\
\hline Utilidade percebida & $\begin{array}{l}\text { O grau em que uma pessoa verifica que a adoção de um produto ou } \\
\text { serviço com atributos tecnológicos lhe trará uma vantagem latente para } \\
\text { execução das atividades do seu cotidiano, quando comparado a uma } \\
\text { tecnologia já existente. }\end{array}$ & Gao e Bai (2014) \\
\hline
\end{tabular}

H1 - A utilidade percebida é determinante para a adoção de produtos baseados na "Internet das Coisas" por parte dos consumidores.

\section{Facilidade de uso produto ou serviço com atributos tecnológicos. Se a utilização de tal percebida $\quad$ produto ou serviço for demasiado complexa, os consumidores poderão demonstrar resistência em adotá-lo, e também poderão não encontrar utilidade no mesmo.}

H2 - A facilidade de uso percebida é determinante para a adoção de produtos baseados na "Internet das Coisas" por parte dos consumidores.

H3 - A variável facilidade de uso impacta positivamente a variável utilidade percebida

Influência Social

O grau em que o consenso das pessoas próximas ao convívio social de um indivíduo, o influencia ou o encoraja em favor da adoção de um Gao e Bai (2014) produto ou serviço com os atributos tecnológicos.

H4 - A variável Influência social é determinante para a adoção de produtos baseados na "Internet das Coisas" por parte dos consumidores.

Redução da sensação de insegurança e dúvida quanto à qualidade e bom funcionamento do produto, além de aspectos relativos à Gao e Bai (2014) e integridade pessoal de um indivíduo e ao senso de domínio que este Al-Momani, possui sobre suas informações particulares. Os consumidores poderão Mahmoud demonstrar resistência em adotar um produto se este não for confiável Ahmad (2016) e também poderão não encontrar utilidade no mesmo.

H5 - A variável confiança é determinante para a adoção de produtos baseados na "Internet das Coisas" por parte dos consumidores.

H6 - A variável confiança impacta positivamente a variável utilidade percebida

Prazer percebido

O grau em que um indivíduo percebe que a utilização de um produto ou serviço com atributos tecnológicos aumentará a sua sensação de Gao e Bai (2014) bem-estar e contentamento. 
H7 - A variável prazer percebido é determinante para a adoção de produtos baseados na "Internet das Coisas” por parte dos consumidores.

\begin{tabular}{|l|l|l|}
$\begin{array}{l}\text { Controle } \\
\text { comportamental } \\
\text { percebido }\end{array}$ & $\begin{array}{l}\text { Sensação de aptidão para utilizar um serviço ou produto com atributos } \\
\text { tecnológicos, além do domínio e direção das funções de tal produto. }\end{array}$ & $\begin{array}{l}\text { Gao e } \\
\text { Al-Momani, } \\
\text { Mahmoud e } \\
\text { Ahmad (2016) }\end{array}$ \\
\hline
\end{tabular}

H8 - A variável controle comportamental percebido é determinante para a adoção de produtos baseados na "Internet das Coisas" por parte dos consumidores.

\begin{tabular}{|l|l|l|}
\hline Custo & $\begin{array}{l}\text { A quantia de dinheiro necessária a ser desembolsada para adquirir um } \\
\text { produto ou serviço com atributos tecnológicos. Se o preço a ser pago } \\
\text { for muito maior do que aquele que um indivíduo pode pagar, este } \\
\text { tenderá a resistir à adoção deste produto ou serviço. }\end{array}$ & $\begin{array}{l}\text { Al-Momani, } \\
\text { Ahmad (2016) }\end{array}$ \\
\hline $\begin{array}{l}\text { H9 - A variável custo é determinante para a adoção de produtos baseados na "Internet das Coisas" por parte dos } \\
\text { consumidores. }\end{array}$ \\
\hline
\end{tabular}

Quadro 1 - Variáveis influenciadoras do processo de adoção de produtos com atributos da "Internet das Coisas".

Fonte: elaboração própria.

Considerando as variáveis acima definidas, este estudo buscou verificar quais delas são determinantes para a adoção dos chamados "Objetos Inteligentes" por parte dos consumidores no contexto brasileiro. Os procedimentos metodológicos utilizados para solucionar este questionamento seguem detalhados na próxima seção.

\section{PROCEDIMENTOS METOdOLÓGICOS}

Este estudo tem cunho exploratório, uma vez que objetivou perscrutar um tema específico explorando-o a fim de compreendê-lo melhor e alcançar respostas para os questionamentos propostos pela pesquisa (Migueles, 2004), e quantitativo, pois visou à mensuração e ponderação de dados, por meio de recursos estatísticos (Prodanov \& Freitas, 2013).

O procedimento de pesquisa adotado foi a pesquisa de tipo survey, por meio da aplicação de um questionário construído a partir do site Survey Monkey, o qual ficou disponível de 20/03/2017 até $29 / 04 / 2017$, tendo como objeto os brasileiros que acessam a internet, não havendo nenhum outro critério além deste.

Levando-se em conta que atualmente, no Brasil, aproximadamente 102 milhões de pessoas acessam a internet regularmente (Portal Brasil, 2016, 13 de Setembro), a amostra não probabilística simples considerada foi de 208 pessoas (cálculo amostral com nível de confiança de 95\%), levando- se em conta ainda a constatação de Malhotra (2012) de que, para pesquisas dessa natureza é necessário considerar uma amostra maior do que 200. O link da pesquisa foi enviado por e-mail, e compartilhado em redes sociais, em grupos de discussão de tecnologia disponíveis no Facebook, e também através do aplicativo de mensagens Whatsapp.

O questionário aplicado contou com um vídeo explicativo que teve como objetivo mostrar o que é um objeto inteligente e como a "internet das coisas" se aplica nesse tipo de dispositivo, de maneira didática, resumida e aplicada no dia-a-dia. Este vídeo mostrou como a "internet das coisas" já está presente na vida de certos consumidores, e já disponíveis para compra. Além disso, para reforçar a compreensão do tema, após o vídeo, o questionário contou também com um pequeno texto que apresentou o conceito e aplicações básicas de "Objetos Inteligentes". Em seguida, 23 questões referentes às variáveis na adoção de novas tecnologias conforme Quadro 1, com o objetivo de avaliar a influência de cada uma delas na adoção de "Objetos Inteligentes" por parte dos consumidores pesquisados. Para cada uma das questões, foi possível selecionar dos números 1 a 5 , onde 1 significava "discordo totalmente" e 5 "concordo totalmente", conforme escala Likert de 5 pontos. Por fim, o questionário contou também com perguntas gerais sobre gênero, faixa etária, formação e renda dos respondentes.

Um pré-teste foi aplicado numa amostra de 30 pessoas validando o questionário a ser aplicado. Por fim, os dados obtidos do questionário oficial 
foram analisados através de um instrumento de estatística multivariada, a saber, Modelagem de Equações Estruturais (MEE) a partir do software SmartPLS 3 através da modelagem de caminho (path modeling). A utilização desse método de análise (MEE) justifica-se pela sua robustez e processo simplificado de verificar e validar o vínculo existente entre inúmeras variáveis, minimizando possíveis incoerências entre elas, o que explica a sua crescente aplicação em pesquisas na área de Marketing para validação de modelos teóricos (Hair, Gabriel \& Patel, 2014). Nesse sentido, foi possível verificar a influência das variáveis independentes pontuadas no Quadro 1 sobre a variável dependente "intenção de uso". A análise dos resultados encontrados segue na próxima seção.

\section{ANÁLISE DE RESULTADOS}

\subsection{Caracterização da Amostra}

Como ponto de partida da análise dos resultados, fez-se necessário verificar os dados gerais concernentes ao perfil dos respondentes. No que diz respeito ao gênero, $53 \%$ dos respondentes eram do sexo feminino e $47 \%$ do sexo masculino. A idade média da amostra foi de 31 anos de idade, a mediana 28 anos e a moda 21 anos de idade. No que tange à formação acadêmica dos respondentes, a maioria $(37 \%)$ possui o ensino médio completo, seguida de $36 \%$ com nível de ensino superior, $7 \%$ com nível de ensino fundamental, e $20 \%$ com especializações como pós-graduação, mestrado, doutorado, MBA, etc. Por fim, no que se refere à renda mensal, $38 \%$ dos respondentes declararam possuir renda de até $\mathrm{R} \$ 1.760,00 ; 29 \%$ de $\mathrm{R} \$$ $1.760,01$ a $\mathrm{R} \$ 3.720,00 ; 25 \%$ de $\mathrm{R} \$ 3.720,01$ a $\mathrm{R} \$ 8.800,00 ; 7 \%$ de $\mathrm{R} \$ 8.800,01$ a $\mathrm{R} \$ 17.600,00$ e apenas $0,5 \%$ de $\mathrm{R} \$ 17.600,01$ ou mais.

\subsection{Validação do Modelo}

Segundo o método proposto por Ringle, Silva e Bido (2014), para análise através da Modelagem de Equações Estruturais, faz-se necessário verificar a confiabilidade do modelo, a validade convergente e a validade discriminante do mesmo. Estes aspectos visam identificar, respectivamente: se existe coerência e solidez no modelo, se realmente existe relação entre os itens teoricamente relacionados no modelo e se as variáveis consideradas não interferem umas nas outras (Oliveira, Marinho \& Dias, 2016).

\subsubsection{Confiabilidade do Modelo}

Segundo Zambaldi, Costa e Ponchio (2014), a confiabilidade composta é o indicador mais adequado para medir a confiabilidade de métodos de análise que consideram a modelagem de caminho que é o caso do PLS-PM (Partial Least Squares Path Modeling) considerado nessa pesquisa, sendo menos sensível a quantidade de indicadores em cada variável. O padrão aceitável para esse indicador é $\mathrm{CC}>0,7$. Uma primeira análise foi feita no software gerando os seguintes resultados:

Tabela 1 - Confiabilidade composta do modelo.

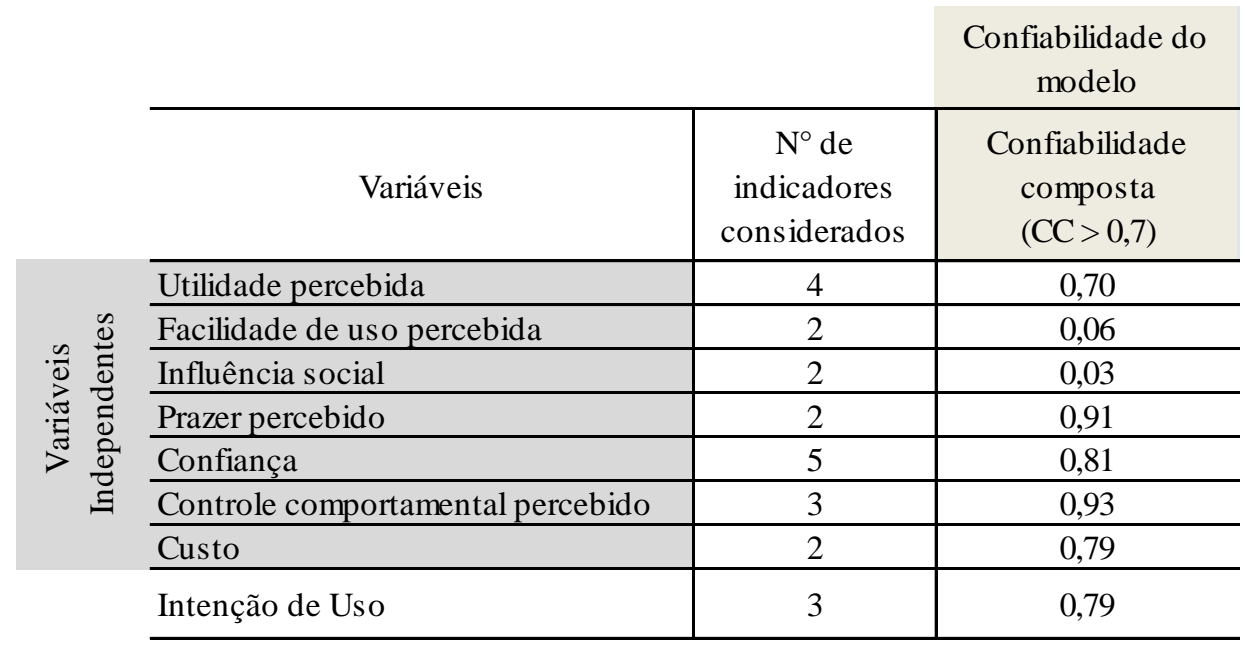

Fonte: elaboração própria.

Os dados acima revelam que as variáveis facilidade de uso e influência social não demonstraram bons índices de confiabilidade composta, e por isso, fez-se necessário ajustar o modelo removendo tais variáveis e certos indicadores das demais, evitando distorções e vieses 
uma vez que o software refaz os cálculos desconsiderando totalmente esses elementos. Após esse ajuste, obteve-se o seguinte cenário confirmando a confiabilidade do modelo ajustado:

Tabela 2 - Confiabilidade composta do modelo ajustado.

\begin{tabular}{|c|c|c|c|}
\hline & Variáveis & $\begin{array}{c}\mathrm{N}^{\circ} \mathrm{de} \\
\text { indicadores } \\
\text { considerados }\end{array}$ & $\begin{array}{c}\text { Confiabilidade } \\
\text { composta } \\
(\mathrm{CC}>0,7) \\
\end{array}$ \\
\hline \multirow{6}{*}{ 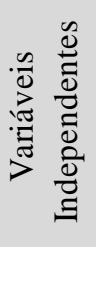 } & Utilidade percebida & 2 & 0,84 \\
\hline & Prazer percebido & 2 & 0,91 \\
\hline & Confiança & 4 & 0,82 \\
\hline & Controle comportamental percebido & 3 & 0,93 \\
\hline & Custo & 2 & 0,80 \\
\hline & Intenção de Uso & 3 & 0,79 \\
\hline
\end{tabular}

Fonte: elaboração própria.

\subsubsection{Validade Convergente do Modelo}

Ringle et al. (2014) recomendam com base no critério Fornell e Larcker, que para garantir que haja validade convergente, isto é, que as relações teoricamente estabelecidas sejam realmente válidas, as AVEs (Avarage Variance Extracted) devem ser > 0,5. A Tabela 3 indica que existe validade convergente entre os indicadores e as variáveis latentes do modelo ajustado, pois todas as AVEs são $>0,5$.

Tabela 3 - Resultados do cálculo de validade convergente (AVE).

\begin{tabular}{|c|c|c|c|}
\hline & Variáveis & $\begin{array}{c}\mathrm{N}^{\circ} \mathrm{de} \\
\text { indicadores } \\
\text { considerados }\end{array}$ & $\begin{array}{c}\mathrm{AVE} \\
(\mathrm{AVE}>0,5)\end{array}$ \\
\hline \multirow{6}{*}{ 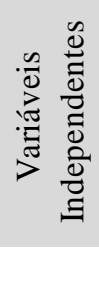 } & Utilidade percebida & 2 & 0,73 \\
\hline & Prazer percebido & 2 & 0,84 \\
\hline & Confiança & 4 & 0,55 \\
\hline & Controle comportamental percebido & 3 & 0,81 \\
\hline & Custo & 2 & 0,66 \\
\hline & Intenção de Uso & 3 & 0,57 \\
\hline
\end{tabular}

Fonte: elaboração própria.

\subsubsection{Validade Discriminante do Modelo}

Confirmada a validade convergente, verificou-se a validade discriminante do modelo, garantindo que as variáveis não possuem correlação muito alta entre si interferindo na distinção de cada uma (Hair, Hult, Ringle \& Sarstedt, 2014). Para tanto, os valores de AVE devem ser menores do que a raiz quadrada da correlação total existente em cada ligação do modelo mostrado na Figura 2. Os valores da Tabela 4 comprovam a validade discriminante do modelo. 
Tabela 4 - Resultados do cálculo de validade discriminante.

\begin{tabular}{l|c|c|c|c|c|c}
\hline \multicolumn{1}{c|}{ Variáveis } & Confiança & $\begin{array}{c}\text { Controle } \\
\text { comportamental } \\
\text { percebido }\end{array}$ & Custo & $\begin{array}{c}\text { Intenção } \\
\text { de uso }\end{array}$ & $\begin{array}{c}\text { Prazer } \\
\text { percebido }\end{array}$ & $\begin{array}{c}\text { Utilidade } \\
\text { percebida }\end{array}$ \\
\hline Confiança & $\mathbf{0 , 7 4}$ & & & & & \\
\hline Controle comportamental percebido & 0,28 & $\mathbf{0 , 9 0}$ & & & & \\
\hline Custo & 0,13 & 0,46 & $\mathbf{0 , 8 1}$ & & & \\
\hline Intenção de uso & 0,22 & 0,62 & 0,62 & $\mathbf{0 , 7 6}$ & & \\
\hline Prazer percebido & 0,26 & 0,39 & 0,53 & 0,46 & $\mathbf{0 , 9 2}$ & \\
\hline Utilidade percebida & $-0,06$ & $-0,32$ & 0,01 & $-0,22$ & 0,06 & $\mathbf{0 , 8 6}$ \\
\hline
\end{tabular}

Fonte: elaboração própria.

\subsection{Avaliação do Modelo Estrutural}

Depois de validar o modelo, faz-se necessário avaliar a estrutura do mesmo analisando o quanto as variáveis consideradas conseguem explicar a intenção de uso de "Objetos Inteligentes". Isso deve ser feito através do indicador $\mathrm{R}^{2}$ tendo em mente que $\mathrm{R}^{2}=2 \%$ significa efeito pequeno, $\mathrm{R}^{2}=$ $13 \%$ efeito médio e $\mathrm{R}^{2}=26 \%$ efeito grande. Além disso, é importante identificar "quanto o modelo se aproxima do que se esperava dele (ou a qualidade da predição do modelo ou acurácia do modelo ajustado)." (Ringle et al., 2014, p.68) através do indicador $\mathrm{Q}^{2}$ que deve ser > 1 . Os resultados da Tabela 5 indicam que $55 \%$ da intenção de uso de "Objetos Inteligentes" da amostra pesquisada pode ser explicada pelas variáveis consideradas (confiança, utilidade percebida, controle comportamental, custo e prazer percebido) tendo um efeito grande. Porém não se verificou o mesmo entre as variáveis confiança e utilidade percebida. No que diz respeito à qualidade de predição do modelo, os resultados se demonstraram maiores que zero, indicando certo nível de precisão considerando a variável intenção de uso.

Tabela 5 - Resultados do cálculo de avaliação do modelo estrutural ( $\mathrm{R}^{2}$ e $\left.\mathrm{Q}^{2}\right)$.

\begin{tabular}{l|c|c}
\cline { 2 - 3 } \multicolumn{2}{c|}{$\mathbf{R}^{\mathbf{2}}$} & $\mathbf{Q}^{\mathbf{2}}$ \\
\hline Intenção de uso & 0,548 & 0,134 \\
\hline Utilidade percebida & 0,004 & $-0,010$ \\
\hline
\end{tabular}

Fonte: elaboração própria.

Em seguida, deu-se a análise dos coeficientes estruturais (coeficientes de caminho), para então prosseguir com a confirmação das hipóteses. Para tanto, foi necessário verificar qual o efeito da relação entre as variáveis independentes e a variável dependente analisada (intenção de uso), qual o tamanho desse efeito, e a significância. A tabela 6 mostra os resultados que serão discutidos mais a frente na seção de confirmação das hipóteses.

Tabela 6 - Análise dos coeficientes de caminho.

\begin{tabular}{l|c|c|c|c}
\hline \multicolumn{1}{c|}{ Correlações } & $\begin{array}{c}\text { Efeito } \\
\text { total }\end{array}$ & $\begin{array}{c}\text { Tamanho do } \\
\text { efeito (f }{ }^{2}\end{array}$ & $\begin{array}{c}\text { Clas sificação do } \\
\text { efeito } \\
\text { (HAIR } \text { et al, 2014) }\end{array}$ & $\begin{array}{c}\text { Significância } \\
(\mathrm{p}<0,05)\end{array}$ \\
\hline Confiança -> Intenção de uso & 0,05 & 0,003 & Pequeno & 0,406 \\
\hline Confiança -> Utilidade percebida & $-0,06$ & 0,004 & Pequeno & 0,366 \\
\hline Controle comportamental percebido -> Intenção de uso & 0,35 & 0,165 & Médio & 0,049 \\
\hline Custo -> Intenção de uso & 0,40 & 0,226 & Médio & 0,046 \\
\hline Prazer percebido -> Intenção de uso & 0,10 & 0,015 & Pequeno & 0,292 \\
\hline Utilidade percebida -> Intenção de uso & $-0,12$ & 0,027 & Pequeno & 0,079 \\
\hline
\end{tabular}

Fonte: elaboração própria. 
O modelo estrutural final ajustado com seus respectivos resultados de correlação entre cada variável segue detalhado na Figura 2.

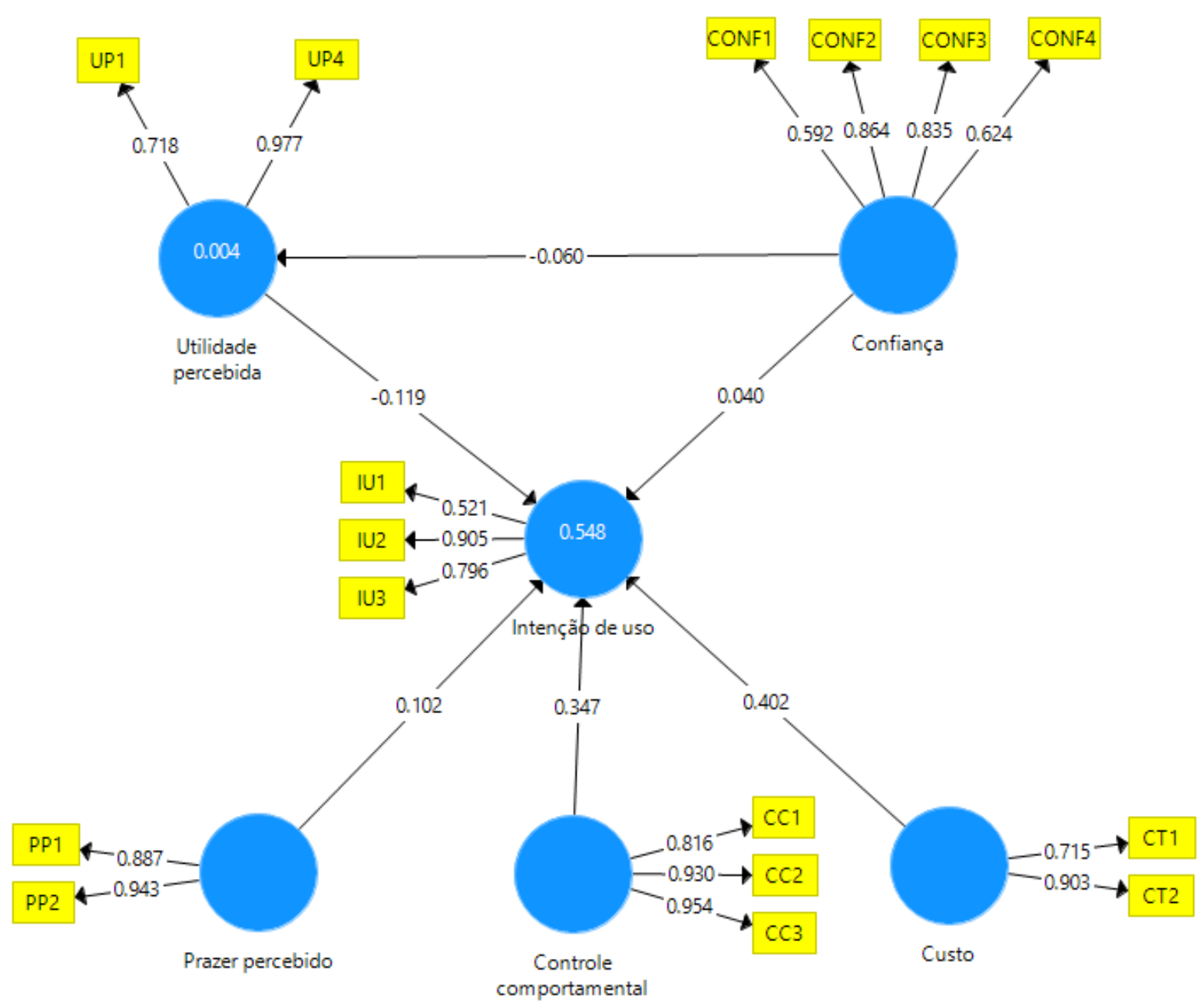

Figura 2 - Modelo estrutural ajustado.

Fonte: elaboração própria.

\subsection{Confirmação das Hipóteses}

A partir da análise dos resultados da Tabela 6, verificou-se que a utilidade percebida não possui influência positiva sobre a intenção de uso (efeito total $=-0,12)$, negando a H1. O construto facilidade de uso não demonstrou bons índices de confiabilidade composta $(\mathrm{CC}=0,06)$ e por isso foi removido do modelo proposto, negando a $\mathrm{H} 2$. Por conseguinte, a H3 também não foi confirmada, devido à exclusão da variável facilidade de uso. Do mesmo modo, a variável influência social não apresentou um índice aceitável de confiabilidade composta $(\mathrm{CC}=0,03)$ sendo igualmente excluída do modelo, negando a $\mathrm{H} 4$.
A variável confiança possui um efeito total (beta) de 0,05 sobre a variável intenção de uso, o que significa que embora exista relação entre elas, esta é muito pequena e não possui significância. Além disso, não foi verificada influência positiva entre a variável confiança e a variável utilidade percebida (beta $=-0,06$ ). Desse modo, H5 e H6 também não foram confirmadas.

A variável prazer percebido possui influência positiva sobre a intenção de uso (beta $=$ 0,10), porém essa influência tem efeito e significância pequenos, o que nega a H7. Por outro lado, a variável controle comportamental percebido, além de possuir influência positiva (beta $=0,35$ ) sobre a intenção de uso, possui um efeito médio ( $\mathrm{f}^{2}$ 
$=0,165)$ e significativo $(\mathrm{p}<0,05)$, confirmando a $\mathrm{H} 8$. De igual modo, a variável custo apresenta influência positiva sobre a intenção de uso (beta $=0,40$ ) e o efeito da primeira sobre a segunda é médio, porém muito próximo ao grande $\left(\mathrm{f}^{2}=0,226\right)$, sendo essa relação significante $(\mathrm{p}<0,05)$ confirmando, portanto, a H9.

\section{CONCLUSÃO}

Esta pesquisa teve como objetivo verificar as variáveis determinantes para a adoção de “Objetos Inteligentes" por parte dos consumidores no contexto brasileiro, considerando que a "Internet das Coisas" já tem proporcionado o crescimento da oferta de objetos como esse no mercado de tecnologia brasileiro (Portal Brasil, 2017, 16 de Fevereiro). Para tanto, foram apresentados os conceitos e definições dos termos "Internet das Coisas" e "Objetos Inteligentes" e, por meio de revisão teórica, foram identificados as principais variáveis consideradas pelos consumidores ao adotarem inovações tecnológicas. Em seguida, as hipóteses desse estudo foram expostas e os procedimentos metodológicos utilizados foram detalhados. Por fim, deu-se a análise dos resultados através da Modelagem de Equações Estruturais (MEE) por meio do software SmartPLS 3, comprovando-se a confiabilidade, validade convergente e validade discriminante do modelo. Desse modo foi possível por fim, confirmar ou não as hipóteses inicialmente levantadas.

Como resposta a problemática da pesquisa, verificou-se que, na amostra pesquisada, existe grande propensão para adotar "Objetos Inteligentes" $\left(\mathrm{R}^{2}=55 \%\right)$. Nesse contexto, as variáveis custo e controle comportamental percebido se mostraram determinantes na perspectiva dos respondentes. Com relação à variável custo, pode-se afirmar que, ao adquirir um objeto inteligente, os consumidores levarão em conta o seu preço avaliando se os atributos desse produto compensam o seu custo. Se este for demasiadamente caro provavelmente haverá certa resistência em adquiri-lo, isto porque, como se trata de um produto novo, existe certa aversão ao risco de pagar um preço muito alto em um produto ainda pouco conhecido pelos consumidores (AlMomani, Mahmoud \& Ahmad, 2016). Considerando que a maioria dos respondentes (64\%) possui uma renda de até $\mathrm{R} \$ 3.720,00$, essa constatação pode ser ainda melhor compreendida. Desse modo, as empresas produtoras de "Objetos Inteligentes" devem considerar a disponibilidade de versões mais baratas e acessíveis desses produtos para atingir um maior número de pessoas.

Quanto à variável controle comportamental percebido percebe-se que o senso de habilidade e da posse de conhecimentos e aptidões necessários, além do sentimento de poder estar no controle ao utilizar um objeto inteligente, influenciam a adoção desses produtos por parte dos consumidores, confirmando a constatação de Gao e Bai (2014). Nesse sentido, é importante que as empresas reforcem esse sentimento nos consumidores divulgando com detalhes as funcionalidades e atributos dos produtos, e capacitando-os através de tutoriais de como utilizálos. Os dados da presente pesquisa indicam que, uma vez que considerem ter o conhecimento necessário, os consumidores estarão mais propensos a adquirir um objeto inteligente.

As demais variáveis propostas pelo referencial teórico não se demonstraram significativos estatisticamente para a amostra pesquisada, o que implicou em concluir que elas não podem ser consideradas como determinantes.

Esta pesquisa contribui para o campo de estudos do comportamento do consumidor na área de Marketing bem como para a área de Administração, na medida em que abre caminho em uma área de pesquisa ainda pouco explorada no Brasil, como se percebe pela baixa quantidade de estudos com foco no consumidor brasileiro no contexto emergente da "Internet das Coisas" no Brasil.

Os resultados encontrados podem nortear as organizações, desde multinacionais até startups, auxiliando-as na criação de estratégias que fomentem a adoção de "Objetos Inteligentes" baseando-se nas variáveis já comprovadas como determinantes, custo e controle comportamental percebido.

A maioria dos estudos sobre "Internet das Coisas" no Brasil, diz respeito a questões estruturais e técnicas de adequação aos novos padrões necessários para efetividade dessa revolução tecnológica. Porém, como um ponto de partida, o presente estudo auxilia no entendimento de como se dará a reação do consumidor quando a hiperconexão através dos objetos realmente se estabelecer no país, e como as organizações deverão responder a esse cenário.

Considerando que esta pesquisa foi realizada em um período específico, através de um corte no tempo, faz-se necessário que ela seja reproduzida em um corte longitudinal, a fim de verificar se há alteração nos resultados. Além disso, outras pesquisas podem ser realizadas considerando um número maior de indicadores em cada variável ou incluindo novas variáveis, o que permitirá uma análise mais detalhada. Pesquisar amostras de outras regiões do Brasil, bem como em outras realidades demográficas e psicográficas, também possibilitará um aprofundamento maior no tema e permitirá o enriquecimento do conhecimento na área de comportamento do consumidor no contexto da "Internet das Coisas", uma vez que o presente estudo 
pretendeu dar uma visão geral e incidental do assunto.

\section{REFERÊNCIAS}

Al-Momani, A. M., Mahmoud, M. A., \& Sharifuddin, M. (2016). Modeling the adoption of internet of things services: A conceptual framework. IJAR, 2(5), 361-367.

Atzori, L., Iera, A., \& Morabito, G. (2014). From "smart objects" to "social objects": the next evolutionary step of the internet of things. IEEE Comunications Magazine,52(1), 97-105.

Bergamo, F. (2017). O Marketing de relacionamento revisitado: as relações comprador vendedor no contexto da cibercultura. Tese de doutorado. Universidade Federal da Bahia, Salvador, Brazil.

Gao, L., \& Bai, X. (2014). A unified perspective on the factors influencing consumer acceptance of internet of things technology. Asia Pacific Journal of Marketing and Logistics, 26(2), 211-231.

Gubbi, J., Buyya, R., Marusic, S., \& Palaniswami, M. (2013). Internet of Things (IoT): A vision, architectural elements, and future directions. Future generation computer systems, 29(7), 1645-1660.

Hair, J. F., Gabriel, M. L. D. D. S., \& Patel, V. K. (2014). Modelagem de Equações Estruturais Baseada em Covariância (CB-SEM) com o AMOS: Orientações sobre a sua aplicação como uma Ferramenta de Pesquisa de Marketing. REMark, 13(2), 43.

Hair, J.F.; Hult, T.M.; Ringle, C.M. e Sarstedt, M. (2014). A Primer on Partial Least Squares Structural Equation Modeling (PLS-SEM). Los Angeles: SAGE.

Isaia, D. (2016, 15 de Julho). "Internet das Coisas" domina debates em fórum internacional sobre software livre. Recuperado em 21 agosto, 2016, do: http://agenciabrasil.ebc.com.br/pesquisa-einovacao/noticia/2016-07/internet-das-coisasdomina-debates-em-forum-internacional-sobre.

Kadow, A., \& Camargo, C. (2016). Internet das Coisas: Vulnerabilidade, Privacidade e Pontos de Segurança. Revista Competência, 9(1), 153-161.

Lee, I., \& Lee, K. (2015). The Internet of Things (IoT): Applications, investments, and challenges for enterprises. Business Horizons, 58(4), 431-440.
Lemos, A. (2013). A comunicação das coisas: teoria ator-rede e cibercultura. São Paulo: Annablume.

Malhotra, N. K. (2012). Pesquisa de marketing: uma orientação aplicada (5a ed.). Porto Alegre: Bookman.

Migueles, C. (2004). Pesquisa: por que administradores precisam entender disso? Rio de Janeiro: E-Papers.

Oliveira, R. R., Marinho, A., Finelli, M., \& Dias, A. T. (2016). Um Estudo Sobre a Utilização da Modelagem de Equações Estruturais na Produção Científica nas Áreas de Administração e Sistemas de Informação. Brazilian Journal of Management/Revista de Administração da UFSM, 9(4), 559-578.

Paes, W. M. (2014). Interoperabilidade móvel: a Internet das Coisas. Revista da Universidade Vale do Rio Verde, 12(1), 794-810.

Portal Brasil (2017, 16 de fevereiro). Brasil já tem 20 milhões de conexões inteligentes entre máquinas. Recuperado em 22 maio, 2017, do: http://www.brasil.gov.br/ciencia-etecnologia/2017/02/brasil-ja-tem-20-milhoes-deconexoes-inteligentes-entre-maquinas.

Portal Brasil (2016, 13 de Setembro). Pesquisa revela que mais de 100 milhões de brasileiros acessam a internet. Recuperado em 16 fevereiro, 2017, do: http://www.brasil.gov.br/ciencia-etecnologia/2016/09/pesquisa-revela-que-mais-de100-milhoes-de-brasileiros-acessam-a-internet.

Prodanov, C. C., \& de Freitas, E. C. (2013). Metodologia do trabalho científico: métodos e técnicas da pesquisa e do trabalho acadêmico ( $2 \mathrm{a}$ ed.). Novo Hamburgo: Feevale.

Ringle, C. M., Silva, D., \& Bido, D. D. S. (2014). Modelagem de equações estruturais com utilização do SmartPLS. REMark, 13(2), 54-71.

Santaella, L., Gala, A., Policarpo, C., \& Gazoni, R. (2013). Desvelando a Internet das Coisas. Revista GEMInIS, 1(2), 19-32.

Serviço Brasileiro de Apoio às Micro e Pequenas Empresas - SEBRAE (2016, 05 de Janeiro). A Internet das Coisas" está em transição de um mero conceito acadêmico para a realidade empresarial. Recuperado em 21 agosto, 2016, do: http://www.sebrae.com.br/sites/PortalSebrae/artigos /inovacao-e-tecnologia-internet-das- 
coisas, 05d99e665b182410VgnVCM100000b27201 0aRCRD.

Segura, C., \& Hildebrand, H. R. (2014). A Internet das Coisas e os novos paradigmas do consumo. Anais da I Jornada Internacional GEMInIS: Entretenimento Transmídia. São Carlos, SP, Brasil.

Singer, T. (2013). Casas, Carros e Cidades Inteligentes: um estudo do enquadramento midiático da Internet das Coisas. Revista GEMInIS, 1(2), 57-74.
Squirra, S. C. (2016). A tecnologia e a evolução podem levar a comunicação para a esfera das mentes/Technology and evolution may lead communication to the mind sphere. Revista FAMECOS, 23(1), 1-18.

Zambaldi, F., Costa, F. J., \& Ponchio, M. C. (2014). Mensuração em marketing: estado atual, recomendações e desafios. REMark, 13(2), 1-27. 